\title{
Zero-bias conductance anomaly of a FeO-bound Au atom triggered by $\mathrm{CO}$ adsorption
}

\author{
Emile D. L. Rienks, ${ }^{1}$ Niklas Nilius, ${ }^{1, *}$ Hans-Joachim Freund, ${ }^{1}$ Livia Giordano, ${ }^{2}$ \\ Gianfranco Pacchioni, ${ }^{2}$ and Jacek Goniakowski ${ }^{3}$ \\ ${ }^{1}$ Department of Chemical Physics, Fritz-Haber Institut der Max-Planck Gesellschaft, Faradayweg 4-6, D-14195 Berlin, Germany \\ ${ }^{2}$ Dipartimento di Scienza dei Materiali, Università di Milano-Bicocca, via Cozzi, 53-20125 Milano, Italy \\ ${ }^{3}$ Institut des Nanosciences de Paris, UMR 7588 CNRS, Université Paris 6, Campus de Boucicaut, 140 Rue de Lourmel, \\ 75015 Paris, France
}

(Received 10 November 2008; revised manuscript received 8 December 2008; published 17 February 2009)

\begin{abstract}
Single gold-carbonyl species adsorbed on a FeO thin film on $\mathrm{Pt}(111)$ exhibit a pronounced zero-bias conductance anomaly in scanning tunneling spectroscopy, while bare Au atoms reveal a smooth conductance behavior. The phenomenon is attributed to the Kondo effect, which seems to be triggered by AuCO attachment to the oxide surface but originates in fact from the interplay between the Fe magnetic moments and the Pt conduction electrons. This interpretation is supported by the observation of a similar conductance anomaly even for the pristine oxide film under certain circumstances. Apparently, the $\mathrm{FeO} / \mathrm{Pt}(111)$ system is intrinsically susceptible to a Kondo-type behavior, which needs however to be initiated by a small, e.g., adsorbate-induced modification of its magnetic structure. The role of the AuCO in triggering the effect is explored by analyzing the geometric and electronic properties of the ad-complex on the $\mathrm{FeO}$ thin film via tunneling spectroscopy and density-functional theory.
\end{abstract}

DOI: 10.1103/PhysRevB.79.075427

PACS number(s): 73.63.-b, 75.20.Hr, 73.20.Hb, 68.37.Ef

\section{INTRODUCTION}

Kondo physics has recently moved into the focus of surface science thanks to the unique possibility to study this magnetic phenomenon at the atomic scale with scanning tunneling microscopy (STM). After the first observation of the Kondo effect for single magnetic adatoms on metal supports, e.g., $\mathrm{Ce}, \mathrm{Co}$, or $\mathrm{Ni}$ on $\mathrm{Ag}(111)$ and $\mathrm{Au}(111),{ }^{1-3}$ a set of subsequent experiments was initiated to investigate similar phenomena for magnetic molecules, ${ }^{4,5}$ ultrasmall metal aggregates, ${ }^{6}$ and semiconductor quantum dots. ${ }^{7}$ The motivation for exploring Kondo physics by means of STM lies not only in the attempt to characterize magnetism at the local scale. Much more, the Kondo phenomenon is exploited to study details of the electronic structure of adsorbate-support complexes, specifically the interaction between localized $d$ or $f$ orbitals of suitable ad-species and bulk/surface states of the metal support. ${ }^{8-10}$ In addition, indirect information is obtained on the energy position of $d$ and $f$ states, which are usually not accessible to tunneling spectroscopy due to their high spatial localization. ${ }^{3,5}$ The exploration of Kondo physics with the STM therefore provides a possibility to illuminate the interrelation between structural, electronic, and magnetic properties at surfaces.

This paper presents a combined STM and densityfunctional theory (DFT) study of a material system that obviously deviates from the typical Kondo systems. The sample consists of a $\mathrm{FeO}$ bilayer supported on a $\mathrm{Pt}(111)$ single crystal. While bulk $\mathrm{FeO}$ (wüstite) has an antiferromagnetic structure due to the parallel alignment of the Fe magnetic moments within (111) planes and an antiparallel stacking of adjacent planes, the magnetic properties of the thin film are not known. The magnetic structure of $\mathrm{FeO} / \mathrm{Pt}(111)$ will, however, arise from the competition between the intralayer coupling of $\mathrm{Fe}$ magnetic moments and their interaction with the conduction electrons of the Pt support underneath.
Depending on the magnitude of the Fe-Pt versus the Fe-Fe coupling strength, two scenarios are conceivable. In the case of strong $\mathrm{Fe}-\mathrm{Pt}$ coupling and sufficient localization of the $\mathrm{Fe}$ moments, each $\mathrm{Fe}$ spin will act individually and spin-flip scattering processes with the Pt electrons and thus Kondo physics might occur. Assuming a dominance of the Fe-Fe coupling instead, the oxide film will change its magnetization only in a collective manner and any local magnetic response as required for the Kondo effect may be inhibited.

In the following work, the properties of the $\mathrm{FeO} / \mathrm{Pt}(111)$ system are probed with differential conductance $(d I / d V)$ spectroscopy in an STM setup. Kondo-type behavior can be identified by a distinct conductance anomaly emerging at zero bias. For the pristine film, such dip in the $d I / d V$ signal was recently observed in a localized region of the coincidence lattice that forms between the $\mathrm{FeO}$ layer and the $\mathrm{Pt}$ support. ${ }^{11} \mathrm{~A}$ similar response is now revealed for other oxide regions too, however, only after the adsorption of goldcarbonyl species. Apparently, the binding of AuCO modifies the $\mathrm{FeO}$ magnetic structure in a way that it becomes susceptible to spin-flip scattering processes with the Pt conduction electrons. The fact that a small and local perturbation at the oxide surface is sufficient to alter the magnetic response of the system suggests the metastable character of the $\mathrm{FeO}$ magnetic ground state. Possible origins for a magnetic reorganization are discussed in the light of spin-polarized DFT $+U$ calculations. Due to limitations of this approach to describe the $\mathrm{FeO} / \mathrm{Pt}$ magnetic structure and the Kondo effect as a many-body excitation, the interpretation of the observed phenomenon has to remain on a rather basic level. The study sheds, however, light into general properties of thin magnetic films of transition-metal oxides on metal supports.

\section{EXPERIMENTAL AND THEORETICAL DETAILS}

The experiments are carried out in an ultrahigh vacuum STM operated at $5 \mathrm{~K}^{12}$ The electronic properties of the 


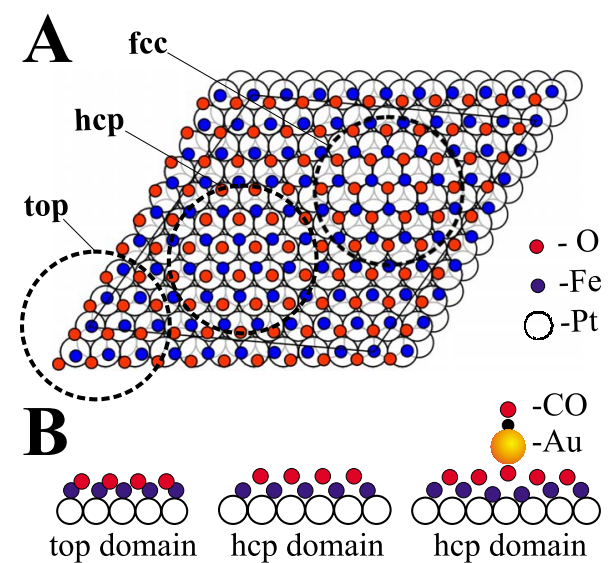

FIG. 1. (Color online) (a) Model of the Moiré cell formed between the FeO bilayer and $\mathrm{Pt}(111)$. (b) Sketch of the local binding configuration of Fe atoms in top and hep domains and of an hcpbound AuCO complex.

sample are derived from differential conductance $(d I / d V)$ spectroscopy, performed with lock-in technique and disabled feed back loop $\left(U_{\text {mod }}=10 \mathrm{mV} \mathrm{rms}, f_{\text {mod }}=900-1100 \mathrm{~Hz}\right)$. According to the Tersoff-Hamann model for vacuum tunneling, the $d I / d V$ signal provides a measure for the local density of sample states (LDOS) ${ }^{13}$ In addition, the second derivative of the tunnel current $\left(d^{2} I / d V^{2}\right)$ is detected in order to identify inelastic contributions to the tunnel current as introduced for instance by vibrational excitations. ${ }^{14}$

The $\mathrm{FeO}$ is prepared by depositing $1 \mathrm{ML}$ iron onto a sputtered/annealed $\mathrm{Pt}(111)$ surface, followed by heating of the sample to $1000 \mathrm{~K}$ in $1 \times 10^{-6} \mathrm{mbar}_{2}$. The resulting film consists of a hexagonal $\mathrm{O}$ layer lying above a hexagonal Fe layer and therefore resembles a single (111) sheet of wüstite bound to the $\operatorname{Pt}(111)$ support. ${ }^{15-17}$ Due to the opposite charges located in the $\mathrm{O}^{\delta-}$ top and the $\mathrm{Fe}^{\delta+}$ interface layer, the film has a polar structure and exposes an out-of-plane surface dipole. The $\mathrm{FeO}$ forms a coincidence lattice with the Pt support, whereby eight $\mathrm{FeO}$ unit cells $\left(d_{\mathrm{Fe}-\mathrm{Fe}}=3.15 \AA\right)$ overgrow nine Pt cells $\left(d_{\mathrm{Pt}-\mathrm{Pt}}=2.78 \AA\right) .{ }^{18}$ The resulting Moiré pattern has a hexagonal structure with $25 \AA$ lattice constant [Fig. 1(a)]. Three domains are distinguishable in the Moiré cell due to their different Fe-Pt stacking configurations. In the top domain, Fe sits on top of the Pt atoms and $\mathrm{O}$ occupies fcc hollow sites, which results in a short Fe-O layer distance and a small surface dipole [Fig. 1(b)]. In fcc (hcp) domains, Fe binds to fcc (hcp) hollow sites, whereas the $\mathrm{O}$ atoms occupy hcp (top) sites of the Pt(111) surface. The hcp regions exhibit the strongest surface dipole due to the large vertical distance between $\mathrm{Fe}$ and $\mathrm{O}$ layers, whereas an intermediate dipole strength is adopted in the fcc domains. ${ }^{19,20}$

In a second preparation step, approximately 0.01 ML of gold is dosed from a resistively heated tungsten filament directly onto the surface held at $10 \mathrm{~K}$. The incoming atoms have sufficient thermal energy to probe a large number of adsorption sites before thermalization and are able to reach their equilibrium binding positions. The Au atoms preferentially adsorb in the hcp domain of the FeO Moiré cell, as this domain exposes the largest surface dipole and enables strong polarization interactions with the adatoms. ${ }^{21}$ This site-
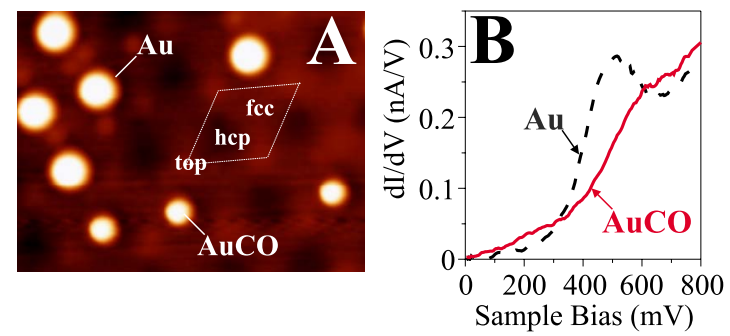

FIG. 2. (Color online) (a) STM topographic image of Au and AuCO species on a thin FeO film on $\mathrm{Pt}(111)\left(13 \times 9 \mathrm{~nm}^{2}, 500 \mathrm{mV}\right.$, $0.1 \mathrm{nA}$ ). (b) Differential conductance spectra of Au and AuCO bound to $\mathrm{FeO}$ hcp regions. The set point for spectroscopy was set with $750 \mathrm{mV}$ and $0.1 \mathrm{nA}$.

specific adsorption leads to the self-assembly of Au atoms into a hexagonal array of $25 \AA$ lattice constant [Fig. 2(a)]. As final step, the $\mathrm{Au} / \mathrm{FeO} / \mathrm{Pt}(111)$ system is exposed to small amounts of $\mathrm{CO}$, whereby the molecules exclusively bind to the $\mathrm{Au}$ adatoms and not on the bare oxide at the given exposure.

Conventional DFT usually fails in describing the electronic structure of transition-metal oxides due to the dominant influence of the on-site Coulomb repulsion between the localized $d$ electrons. We have therefore chosen a spinpolarized $\mathrm{DFT}+U$ approach to describe the $\mathrm{FeO}$ film, as formulated by Dudarev ${ }^{22}$ and implemented in the VASP code. ${ }^{23}$ The calculations use the generalized gradient approximation, the projected augmented wave method, ${ }^{24,25}$ and the PerdewWang 91 functional (400 eV energy cut off). ${ }^{26}$ Similar to earlier studies on $\mathrm{Fe}_{2} \mathrm{O}_{3}$, the Hubbard $U$ parameter and the exchange energy $J$ are set to 4 and $1 \mathrm{eV}$, respectively. ${ }^{27}$ These values provide a satisfactory reproduction of the antiferromagnetic ground state of bulk $\mathrm{FeO}$ and yield a band gap of $1.4 \mathrm{eV}$ (experimental value $2.4 \mathrm{eV}$ ). ${ }^{28,29}$ As the size of the $\mathrm{FeO} / \mathrm{Pt}(111)$ Moiré cell is well beyond the computational capabilities, a simplified nonpseudomorphic cell is constructed by the superposition of a $(\sqrt{ } 7 \times \sqrt{ } 7) R 19^{\circ}-\mathrm{FeO}(111)$ and a $(3 \times 3)-\mathrm{Pt}(111)$ structure. ${ }^{20}$ The computational cell possesses fcc, hcp, and top-bound Fe atoms, reflecting the three stacking configurations of the experimental Moiré structure. The magnetic properties of $\mathrm{FeO}$ are described with an antiferrimagnetic spin alignment that corresponds to the $(\sqrt{3}$ $\times \sqrt{3}) R 30$ collinear equivalent of the $120^{\circ}$-Néel structure and contains $2 / 3$ of cations with one spin orientation and $1 / 3$ with the opposite one. Unsupported FeO slabs with this Néel configuration are found to be lower in energy than their ferromagnetic or row-wise antiferromagnetic counterparts. ${ }^{20}$

\section{RESULTS AND DISCUSSION}

Prior to $\mathrm{CO}$ dosage, single $\mathrm{Au}$ atoms on $\mathrm{FeO} / \mathrm{Pt}(111)$ show up as round protrusions of $1.8 \AA$ apparent height in STM images taken at $0.6 \mathrm{~V}$ sample bias. After exposure, a second Au-related species appears on the surface that is characterized by a $30 \%$ smaller height [Fig. 2(a)]. The new species is assigned to a gold-monocarbonyl (AuCO), formed by the attachment of a single $\mathrm{CO}$ to the adatom. Carbonyl species with more than one $\mathrm{CO}$ ligand are not observed, in ac- 

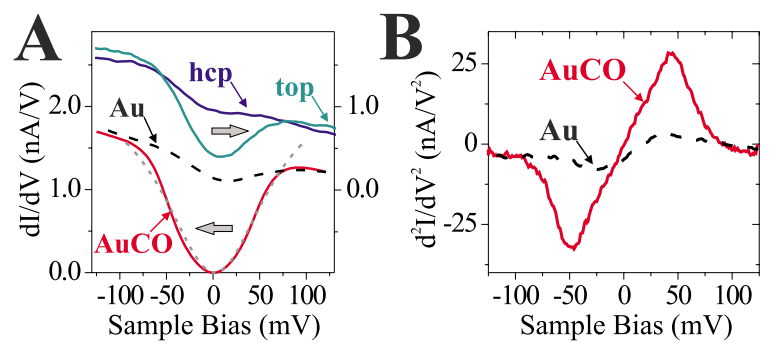

FIG. 3. (Color online) (a) $d I / d V$ spectra taken around the Fermi level of hcp-bound $\mathrm{Au}$ and $\mathrm{AuCO}$ species on $\mathrm{FeO} / \mathrm{Pt}(111)$. The AuCO spectrum was fitted with a Fano line centered at $1 \mathrm{mV}$ and broadened by $112 \mathrm{meV}$ (dotted line). Spectra taken in the hcp and top regions of the pristine oxide are shown for comparison. (b) $d^{2} I / d V^{2}$ spectra of the same Au and AuCO species as in (a) (set point in both cases: $100 \mathrm{mV}$ and $0.1 \mathrm{nA}$ ).

cordance with the monovalent character of gold. The height difference between $\mathrm{Au}$ and $\mathrm{AuCO}$ vanishes at low imaging bias and is traced back to an electronic effect. Single Au atoms on $\mathrm{FeO}$ hcp domains exhibit a distinct peak at $+0.5 \mathrm{~V}$ in the differential conductance that originates from tunneling into the empty Au $6 s$ orbital [Fig. 2(b)]. ${ }^{30}$ In the corresponding AuCO spectra, this peak has vanished and only a monotonously increasing background remains visible, similar to the conductance signature of bare $\mathrm{FeO}$. The removal of unoccupied state density is responsible for the reduced apparent height of the AuCO complex in topographic images taken above $0.5 \mathrm{~V}$ sample bias. According to the DFT calculations, the $\mathrm{Au} 6 s$ shifts to $+3.5 \mathrm{eV}$ upon hybridization with the CO $5 \sigma$ orbital and is not accessible to $d I / d V$ spectroscopy any more. ${ }^{30} \mathrm{~A}$ similar effect has earlier been observed for AuCO complexes bound to $\mathrm{NiAl}(110) .{ }^{31,32}$

The formation of AuCO does not only modify the spectroscopic response in the region of the unoccupied states, but also affects the $d I / d V$ signal directly at the Fermi energy $\left(E_{F}\right)$, as shown in Fig. 3(A). While Au atoms exhibit a smooth conductance behavior at zero bias, a pronounced dip in the conductance is observed for AuCO complexes. In difference spectra $\left(d I / d V_{\mathrm{AuCO}}-d I / d V_{\mathrm{Au}}\right)$, where possible tip effects on the conductance are eliminated, the curve takes the shape of a symmetric antiresonance of $110 \mathrm{mV}$ full width at half maximum (FWHM) centered at $E_{F}$.

In order to decide whether the $d I / d V$ dip originates from a feature in the AuCO LDOS (Ref. 13) or reflects the onset of an inelastic tunneling path associated with a vibrational excitation, ${ }^{14}$ also second-derivative $\left(d^{2} I / d V^{2}\right)$ spectra are acquired [Fig. 3(b)]. In general, vibrational features are identified by their symmetric peak/dip structure appearing at the positive/negative vibrational energy in the $d^{2} I / d V^{2}$ spectra. Although the AuCO spectrum follows this characteristic and exhibits two extreme values located at $\pm(45 \pm 2) \mathrm{mV}$, its detailed shape is in conflict with a true vibrational fingerprint for several reasons. ${ }^{14,31}$ The FWHM of the peak (dip) is with $40 \mathrm{mV}$ too broad to reflect the onset of an inelastic transport channel. For the given sample temperature and modulation bias, the line width should not exceed $15 \mathrm{mV}$ to be compatible with a reasonable vibrational lifetime. ${ }^{33}$ Furthermore, the $d^{2} I / d V^{2}$ spectrum of AuCO shows no plateau region at zero bias, but evolves with constant slope from the dip at

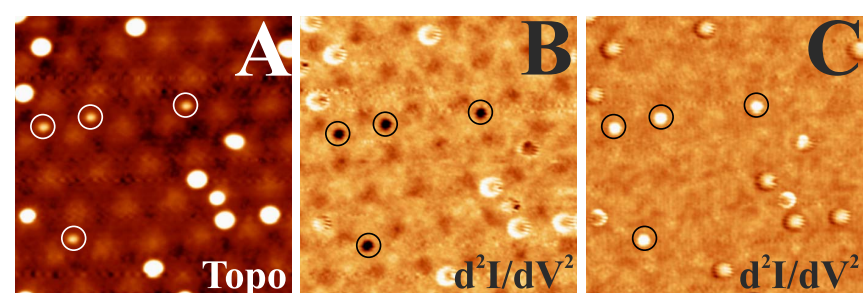

FIG. 4. (Color online) (a) Topography $\left(16.5 \times 16.5 \mathrm{~nm}^{2}, 0.1\right.$ $\mathrm{nA}$ ) and $d^{2} I / d V^{2}$ maps of $\mathrm{CO} / \mathrm{Au} / \mathrm{FeO} / \mathrm{Pt}(111)$ taken at (b) $-30 \mathrm{mV}$ and (c) $+30 \mathrm{mV}$ sample bias. The AuCO species (marked by circles) are clearly identified by a contrast reversal induced by the opposite slope of the $d I / d V$ signal above and below the Fermi level.

negative to the peak at positive bias. In true vibrational spectra, a distinct background feature emerges at $E_{F}$ that marks the reversal of the tunnel current when switching the bias polarity. ${ }^{14}$ Test measurements performed under identical conditions for AuCO on metallic $\mathrm{NiAl}(110)$ revealed the expected $d^{2} I / d V^{2}$ peak-dip structure at $\pm 35 \mathrm{mV}$ with $13 \mathrm{mV}$ linewidth that marks the $\mathrm{CO}$ hindered rotation on the $\mathrm{Au}$ atom. ${ }^{31}$ In the light of these results, we exclude a vibrational origin of the zero-bias conductance anomaly observed on the $\mathrm{FeO}$ film.

The distinct $d^{2} I / d V^{2}$ fingerprint provides, however, a convenient means to discriminate $\mathrm{Au}$ and $\mathrm{AuCO}$ species in spectroscopic maps taken at the peak and dip energies (Fig. 4). The AuCO species are bright in maps taken at $+40 \mathrm{mV}$ due to their $d^{2} I / d V^{2}$ maximum, but appear with inverse contrast at the dip position of $-40 \mathrm{mV}$. This contrast reversal is not observed for the bare adatoms due to their flat secondderivative spectra. The $d^{2} I / d V^{2}$ maps also reveal the high spatial localization of the zero-bias dip within a circle of $4 \AA$ radius around the $\mathrm{AuCO}$.

Excluding a vibrational origin, the observed conductance anomaly at the Fermi level is ascribed to an electronic effect induced by the $\mathrm{Au}-\mathrm{CO}$ bond formation on the $\mathrm{FeO} / \mathrm{Pt}(111)$ surface. In a first attempt, the dip in the $d I / d V$ signal might be explained by a spatially confined decrease in the singleelectron LDOS of the system. ${ }^{13}$ In principle, $\mathrm{CO}$ adsorption could reduce the state density at $E_{F}$, for instance, by repelling $\mathrm{Au}$ or $\mathrm{Pt}(111)$-derived states via Pauli repulsion of the $\mathrm{CO}$ orbitals ${ }^{32,34}$ or bending the $\mathrm{FeO}$ bands in response to a charged ad-species. ${ }^{35,36}$ None of these scenarios is compatible with the state density calculated by DFT. The FeO/ $\mathrm{Pt}(111)$ LDOS is smooth across the Fermi level and reveals no indication for the $\mathrm{FeO}$ bulk band gap due to the strong influence of the metal support [Fig. 5(a)]. Furthermore, neither the Au atom nor the AuCO complex induces states in the vicinity of $E_{F}$ or modify the FeO LDOS in an appropriate way [Fig. 5(b)]. In fact, the lowest Au-induced electronic state is the Au $6 s$ orbital at $+0.5 \mathrm{eV}$, which even shifts upward upon $\mathrm{CO}$ adsorption. Changes in the single-electron LDOS due to adsorption of AuCO can therefore be ruled out as an explanation for the zero-bias dip in the differential conductance.

On the other hand, also quasistates with many-electron character that do not show up in DFT calculations may influence the conductance behavior of an STM junction. A prominent example for a many-body electronic feature is the 


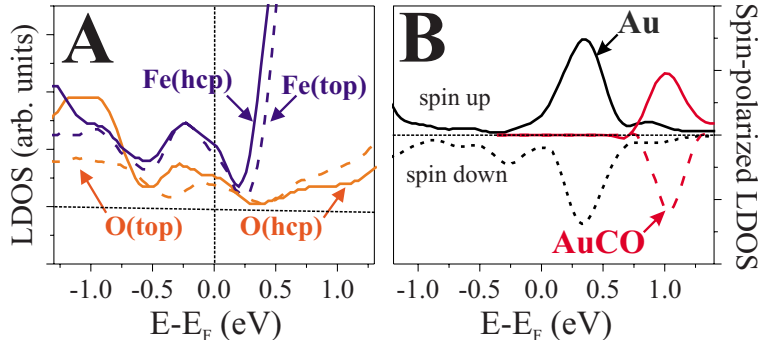

FIG. 5. (Color online) (a) Calculated state density of Fe and O atoms located in hep (solid line) and top regions (dashed line) of the $\mathrm{FeO}$ Moiré cell. No minimum in the single-electron LDOS is observed at the Fermi level. (b) Spin-polarized LDOS of Au and $\mathrm{AuCO}$ species on $\mathrm{FeO} / \mathrm{Pt}(111)$. The resonance of the bare $\mathrm{Au}$ at 0.3 $\mathrm{eV}$ marks the $\mathrm{Au} 6 s$ orbital hybridized with the $\mathrm{FeO}$ states, while the AuCO peak at $1.0 \mathrm{eV}$ has mixed Au $5 d$-CO $2 \pi^{*}$ character. Similar intensities for spin-up and spin-down states (especially below $\left.E_{F}\right)$ indicate the nonmagnetic character of $\mathrm{Au}$ and $\mathrm{AuCO}$.

Kondo resonance that emerges at the Fermi level of diluted magnetic systems. ${ }^{37,38}$ It arises from the hybridization of a localized $d$ or $f$ orbital carrying unpaired electrons and continuum states of the surrounding medium. The localized magnetic moment is quenched by the antiparallel alignment of electron spins in the screening cloud and the resulting many-body state has therefore singlet character. Electron tunneling through such a Kondo resonance results in $d I / d V$ spectra with characteristic Fano shapes at $E_{F} \cdot{ }^{39}$ The Fano line originates from quantum interference effects between a direct tunneling path between tip and sample and an indirect one that involves the Kondo resonance. Depending on the relative weights of both conduction channels, it takes the shape of a symmetric maximum/minimum or an asymmetric peak-dip structure..$^{1-3,40}$ The antiresonance observed here would be compatible with a weak coupling between the tip wave function and a potential Kondo state. The occurrence of Kondo physics is related to a critical temperature $T_{K}$, below which spin-flip scattering processes and the formation of the many-body electronic state are observed. The magnitude of $T_{K}$ that can be determined directly from the Fano line shape ${ }^{41}$ provides hereby a measure for the coupling strength between the magnetic moment and the conduction electrons.

The assignment of the anomalous conductance behavior of $\mathrm{AuCO}$ on $\mathrm{FeO} / \mathrm{Pt}(111)$ to the Kondo effect is plausible for two reasons. First, the system combines the necessary preconditions for Kondo physics, namely, localized Fe magnetic moments that are in contact with the Pt electron gas. And second, a comparable conductance behavior is observed even without $\mathrm{AuCO}$ on the $\mathrm{FeO}$ top domains. ${ }^{11}$ At this particular position of the Moiré cell, a similar antiresonance appears in the $d I / d V$ spectra that is only slightly sharper (FWHM of 86 $\mathrm{meV}$ ) and less pronounced than in the AuCO case [Fig. 3(a)]. The conductance anomaly at the $\mathrm{FeO}$ top domains had previously been associated with the Kondo effect, characterized by a Kondo temperature of $490 \pm 15 \mathrm{~K}$. A similar range of $T_{K}, 650 \pm 10 \mathrm{~K}$, is now determined for the hcp-bound AuCO complex from fitting the $d I / d V$ spectra to the Fano model. ${ }^{41}$ The similarity of the zero-bias $d I / d V$ response in both cases suggests the same physical origin of the phenomenon, being only triggered by different factors.
Once ascribed to the Kondo effect, the role of $\mathrm{Au}+\mathrm{CO}$ adsorption for stimulating this peculiar magnetic response of $\mathrm{FeO} / \mathrm{Pt}(111)$ needs to be analyzed. The $d I / d V$ anomaly is neither observed for pristine $\mathrm{FeO}$ hcp domains, being the preferred $\mathrm{Au}$ binding regions, nor for bare $\mathrm{Au}$ atoms. $\mathrm{CO}$ attachment to an $\mathrm{Au}$ is therefore the necessary precondition for initiating the effect. Two scenarios are conceivable: (i) $\mathrm{CO}$ adsorption creates a magnetic moment in the $\mathrm{Au}$ adatom or (ii) it alters the existing Fe spins below the AuCO in an appropriate way to induce the Kondo response. The first option can be discarded for several reasons. A single Au adatom is not a typical "Kondo candidate," as it has a closed-shell $d^{10}$ configuration and therefore no magnetic moment related to unpaired $d$ or $f$ electrons, in contrast to the typical "Kondo atoms" $\mathrm{Co}, \mathrm{Ni}, \mathrm{Fe}$, or Ce. ${ }^{1,2,9,40} \mathrm{On}$ the other hand, the $\mathrm{Au} 6 s$ orbital carries an unpaired electron in the neutral atom that gives rise to a paramagnetic response $e^{42,43}$ and might be involved in the Kondo effect, too. DFT calculations reveal, however, that an $\mathrm{Au}$ atom on the $\mathrm{FeO}$ hcp domain is positively charged (Au $6 s^{0}$ configuration) due to the transfer of its $6 s$ electron into the Pt support. ${ }^{30}$ The electron transfer is driven by the unusually high work function of the polar $\mathrm{FeO} /$ $\mathrm{Pt}(111)$ system and manifested by the position of the Au 6s-derived state above the Fermi level [Fig. 5(b)]. The spin density at the $\mathrm{Au}$ is not restored upon $\mathrm{CO}$ adsorption, as the $6 s$ orbital shifts even further away from $E_{F}$ and remains unoccupied, while the closed-shell $\mathrm{CO}$ molecule does not introduce a magnetic moment by its own [Fig. 5(b)]. This observation is supported by a number of similar reports in the literature, where $\mathrm{CO}$ is found to maintain or quench but never to increase the spin density of a single adatom. ${ }^{43,44}$ Not surprisingly, no Kondo effect has been detected for AuCO species adsorbed on metal surfaces so far. ${ }^{31}$

As a conclusion, neither the bare $\mathrm{Au}$ nor the $\mathrm{AuCO}$ is able to provide a localized magnetic moment by its own, but contribute to the Kondo effect only by creating a suitable magnetic environment in the FeO/Pt(111) system. Two observations support this interpretation: (i) The zero-bias conductance anomaly appears even without AuCO for the top domains of the FeO Moiré cell [Fig. 3(a)] and (ii) the Kondo temperature observed here is much higher than literature values reported for single magnetic adsorbates on nonmagnetic surfaces, where $T_{K}$ is usually below $100 \mathrm{~K} \cdot{ }^{1-3,9,10}$ A Kondo temperature of around $500 \mathrm{~K}$ is, however, plausible when the magnetic moments are embedded in a two- or threedimensional matrix, as in the $\mathrm{FeO}$ case ${ }^{45}$ Apparently, the $\mathrm{FeO} / \mathrm{Pt}(111)$ system is intrinsically susceptible to Kondo physics, which occurs, however, only in presence of a suitable trigger. Whereas in the $\mathrm{FeO}$ top domains, the local $\mathrm{Fe}-\mathrm{Pt}$ binding configuration seems to create an environment that enables Fe spin-flip scattering events with the Pt conduction electrons, the required stimulus for the hcp domains is provided by the AuCO adsorption. Coming to this conclusion, we have to leave the solid ground of the experimental and theoretical results and can only speculate on the exact interaction mechanism that leads to the Kondo effect. In the final part of the paper, we will therefore discuss three possible scenarios that might explain why the AuCO but not the bare $\mathrm{Au}$ atom is able to modify the magnetic response of the metal/oxide system. 


\section{A. Structural aspects}

The AuCO attachment might influence the local environment of $\mathrm{Fe}$ atoms in a way that they undergo a magnetic restructuring. According to the DFT results, the O-top positions in hcp domains are the preferred binding sites for both the $\mathrm{Au}$ and the AuCO species. The corresponding Au binding energy amounts to $0.7 \mathrm{eV}$, which is $0.2 \mathrm{eV}$ higher than for equivalent sites in top and fcc domains and explains the selfassembly of the ad-atoms. ${ }^{30}$ Due to the positive charge localized at the $\mathrm{Au}$, the $\mathrm{FeO}$ undergoes a local polaronic distortion, in which the $\mathrm{O}$ atom is lifted above the oxygen plane and the three Fe neighbors slightly approach the Pt support, thereby increasing the Fe-O layer distance by $20 \%$ [Fig. 1(b)]. Hence, the Fe ions experience a larger coupling to the $\mathrm{Pt}$ atoms, which is similar to the situation in the top domains that exhibit intrinsic Kondo behavior. Furthermore, the octahedral symmetry of the $\mathrm{Fe}$ ions in the hcp domains $\left(\mathrm{O}_{3}-\mathrm{Fe}-\mathrm{Pt}_{3}\right)$ is partly lifted upon $\mathrm{Au}$ binding, as the distance to one oxygen atom is considerably enlarged. In comparison, the $\mathrm{Fe}$ ions in top domains resume a tetrahedral geometry $\left(\mathrm{O}_{3}-\mathrm{Fe}-\mathrm{Pt}\right)$. Both trends, the reduced Fe-Pt separation and the distorted binding configuration, amplify in the presence of $\mathrm{CO}$, as the AuCO complex carries a higher positive charge and enhances the effect of the polaronic distortion. It seems to be unlikely that the adsorbate-induced $\mathrm{FeO}$ lattice distortion alone is sufficient to induce a change in the magnetic response. Given the exponential dependence of the Kondo temperature on the Fe-Pt interaction strength, ${ }^{37,40}$ it might nonetheless contribute to the observed phenomenon.

\section{B. Change of the Fe magnetic moments}

Also a local alteration of the Fe moments might explain the occurrence of Kondo physics upon AuCO adsorption to the hep domains. The DFT $+U$ calculation predicts a constant magnetic moment of $3.6 \mu_{\mathrm{Bohr}}$ for all $\mathrm{Fe}$ atoms in the pristine film despite of the large differences in their local binding geometry on the Pt support. Not surprisingly, also the adsorption of $\mathrm{Au}$ or $\mathrm{AuCO}$ species to an oxygen ion is unable to alter the magnetic moment of the neighboring Fe ions. A change of the Fe moments due to $\mathrm{AuCO}$ attachment is therefore ruled out as a possible origin for the modified magnetic response.

\section{Change of the long-range magnetic ordering}

Even if the respective moments are constant, their orientational alignment and mutual interaction might be influenced by $\mathrm{AuCO}$ adsorption. Unfortunately, the analysis of such effects is beyond the experimental and theoretical possibilities of this work, as not even the initial spin ordering in the FeO Moiré cell on $\mathrm{Pt}(111)$ is not known. From an experi- mental point of view, only ferromagnetic ordering can be excluded, as no magnetic signal is detected in electronparamagnetic resonance spectroscopy. ${ }^{11} \mathrm{DFT}+U$ calculations on a simplified $\mathrm{FeO}$ superstructure cell suggest that an antiferromagnetic spin alignment, equivalent to the $120^{\circ}$-Néel structure, has the lowest energy out of a limited set of test configurations. ${ }^{20}$ Further details of the magnetic structure are, however, inaccessible due to the size of the $\mathrm{FeO}$ Moiré cell, the threefold symmetry of the system that requires spin frustration, and the possible presence of inplane and out-of-plane spin orientations. Solutions for such complex magnetic networks are spin spirals with variable wave vectors ${ }^{46}$ as identified for $\mathrm{Fe} / \mathrm{Ir}(111)$ and $\mathrm{Mn} / \mathrm{W}(001)$ using spin-polarized STM. ${ }^{47,48}$ The development of a comparably complex magnetic structure is expected for the $\mathrm{FeO} /$ $\mathrm{Pt}(111)$ system, too. Within the magnetic unit cell, only selected Fe spins might be able to perform Kondo-type spinflip scattering with the Pt conduction electrons, while others are tightly embedded in the magnetic network. This kind of susceptible spins seems to be intrinsically present in the $\mathrm{FeO}$ top domains and may form also in the hep domains upon AuCO adsorption. At the present stage, only the AuCOinduced lattice distortion and the charge-impurity created on the $\mathrm{FeO}$ surface are identified as possible triggers for the magnetic restructuring.

\section{CONCLUSION}

A spatially confined antiresonance in the zero-bias conductance has been observed for AuCO complexes adsorbed on a FeO thin film on $\mathrm{Pt}(111)$ using STM. The phenomenon is assigned to the Kondo effect, although no magnetic measurements could be performed to proof this assumption. As a similar conductance anomaly is detected also in the top domains of the pristine $\mathrm{FeO}$ film, we believe that a particular Fe binding geometry is responsible for the Kondo-type response. The required Fe-O configuration is either stabilized by the geometric constraint given by the $\mathrm{Pt}(111)$ support or by a local lattice distortion introduced by the AuCO complex. The gold-carbonyl therefore provides only the external stimulus to modify the $\mathrm{FeO}$ magnetic structure without adding intrinsic magnetic properties by its own. The possibility to induce the Kondo effect in the FeO/Pt(111) system already with a small perturbation suggests the metastable character of its magnetic ground state. Thin magnetic films on metal supports might therefore offer new possibilities to realize switchable magnetic systems with potential applications in microelectronics, magnetic sensors, or storage devices.

\section{ACKNOWLEDGMENT}

The work has been supported by the COST Action D41. 
*Corresponding author; nilius@fhi-berlin.mpg.de

${ }^{1}$ J. T. Li, W. D. Schneider, R. Berndt, and B. Delley, Phys. Rev. Lett. 80, 2893 (1998).

${ }^{2}$ V. Madhavan, W. Chen, T. Jamneala, M. F. Crommie, and N. S. Wingreen, Science 280, 567 (1998).

${ }^{3}$ V. Madhavan, T. Jamneala, K. Nagaoka, W. Chen, Je-Luen Li, Steven G. Louie, and M. F. Crommie, Phys. Rev. B 66, 212411 (2002).

${ }^{4}$ A. Zhao, Q. Li, L. Chen, H. Xiang, W. Wang, S. Pan, B. Wang, X. Xiao, J. Yang, J. G. Hou, and Q. Zhu, Science 309, 1542 (2005).

${ }^{5}$ L. Gao, W. Ji, Y. B. Hu, Z. H. Cheng, Z. T. Deng, Q. Liu, N. Jiang, X. Lin, W. Guo, S. X. Du, W. A. Hofer, X. C. Xie, and H. J. Gao, Phys. Rev. Lett. 99, 106402 (2007)

${ }^{6}$ T. Jamneala, V. Madhavan, and M. F. Crommie, Phys. Rev. Lett. 87, 256804 (2001).

${ }^{7}$ S. M. Cronenwett, T. H. Oosterkamp, and L. P. Kouwenhoven, Science 281, 540 (1998).

${ }^{8}$ V. Madhavan, W. Chen, T. Jamneala, M. F. Crommie, and N. S. Wingreen, Phys. Rev. B 64, 165412 (2001).

${ }^{9}$ P. Wahl, L. Diekhöner, M. A. Schneider, L. Vitali, G. Wittich, and K. Kern, Phys. Rev. Lett. 93, 176603 (2004); P. Wahl, L. Diekhöner, G. Wittich, L. Vitali, M. A. Schneider, and K. Kern, ibid. 95, 166601 (2005).

${ }^{10}$ J. Henzl and K. Morgenstern, Phys. Rev. Lett. 98, 266601 (2007).

${ }^{11}$ E. D. L. Rienks, N. Nilius, L. Giordano, J. Goniakowski, G. Pacchioni, M. P. Felicissimo, T. Risse, H.-P. Rust, and H.-J. Freund, Phys. Rev. B 75, 205443 (2007).

${ }^{12}$ H.-P. Rust, J. Buisset, E. K. Schweizer, and L. Cramer, Rev. Sci. Instrum. 68, 129 (1997).

${ }^{13}$ J. Tersoff and D. R. Hamann, Phys. Rev. Lett. 50, 1998 (1983).

${ }^{14}$ W. Ho, J. Chem. Phys. 117, 11033 (2002).

${ }^{15}$ G. H. Vurens, V. Maurice, M. Salmeron, and G. A. Somorjai, Surf. Sci. 268, 170 (1992).

${ }^{16}$ M. Ritter, W. Ranke, and W. Weiss, Phys. Rev. B 57, 7240 (1998); W. Ranke, M. Ritter, and W. Weiss, ibid. 60, 1527 (1999).

${ }^{17}$ H. C. Galloway, P. Sautet, and M. B. Salmeron, Phys. Rev. B 54, R11145 (1996).

${ }^{18}$ Y. J. Kim, C. Westphal, R. X. Ynzunza, H. C. Galloway, M. B. Salmeron, M. A. Van Hove, and C. S. Fadley, Phys. Rev. B 55, R13448 (1997).

${ }^{19}$ E. D. L. Rienks, N. Nilius, H.-P. Rust, and H.-J. Freund, Phys. Rev. B 71, 241404(R) (2005).

${ }^{20}$ L. Giordano, G. Pacchioni, J. Goniakowski, N. Nilius, E. D. L. Rienks, and H.-J. Freund, Phys. Rev. B 76, 075416 (2007).

${ }^{21}$ N. Nilius, E. D. L. Rienks, H.-P. Rust, and H.-J. Freund, Phys. Rev. Lett. 95, 066101 (2005).

${ }^{22}$ S. L. Dudarev, G. A. Botton, S. Y. Savrasov, C. J. Humphreys, and A. P. Sutton, Phys. Rev. B 57, 1505 (1998).

${ }^{23}$ G. Kresse and J. Hafner, Phys. Rev. B 47, 558 (1993); G. Kresse and J. Furthmüller, ibid. 54, 11169 (1996).
${ }^{24}$ P. E. Blöchl, Phys. Rev. B 50, 17953 (1994).

${ }^{25}$ O. Bengone, M. Alouani, P. E. Blöchl, and J. Hugel, Phys. Rev. B 62, 16392 (2000).

${ }^{26}$ J. P. Perdew, J. A. Chevary, S. H. Vosko, K. A. Jackson, M. R. Pederson, D. J. Singh, and C. Fiolhais, Phys. Rev. B 46, 6671 (1992).

${ }^{27}$ G. Rollmann, A. Rohrbach, P. Entel, and J. Hafner, Phys. Rev. B 69, 165107 (2004).

${ }^{28}$ I. I. Mazin and V. I. Anisimov, Phys. Rev. B 55, 12822 (1997).

${ }^{29}$ Z. Fang, I. V. Solovyev, H. Sawada, and K. Terakura, Phys. Rev. B 59, 762 (1999).

${ }^{30}$ L. Giordano, G. Pacchioni, J. Goniakowski, N. Nilius, E. D. L. Rienks, and H.-J. Freund, Phys. Rev. Lett. 101, 026102 (2008).

${ }^{31}$ T. M. Wallis, N. Nilius, and W. Ho, J. Chem. Phys. 119, 2296 (2003).

${ }^{32}$ M. Persson, Phys. Rev. B 72, 081404(R) (2005).

${ }^{33}$ L. J. Lauhon and W. Ho, Rev. Sci. Instrum. 72, 216 (2001).

${ }^{34}$ L. Bartels, G. Meyer, and K.-H. Rieder, Chem. Phys. Lett. 297, 287 (1998).

${ }^{35}$ R. J. Hamers, J. Vac. Sci. Technol. B 6, 1462 (1988).

${ }^{36}$ M. Batzill, K. Katsiev, D. J. Gaspar, and U. Diebold, Phys. Rev. B 66, 235401 (2002).

${ }^{37}$ J. Kondo, Prog. Theor. Phys. 32, 37 (1964).

${ }^{38}$ A. Shimoyamada, S. Tsuda, K. Ishizaka, T. Kiss, T. Shimojima, T. Togashi, S. Watanabe, C. Q. Zhang, C. T. Chen, Y. Matsushita, H. Ueda, Y. Ueda, and S. Shin, Phys. Rev. Lett. 96, 026403 (2006).

${ }^{39}$ U. Fano, Phys. Rev. 124, 1866 (1961).

${ }^{40}$ V. Madhavan, W. Chen, T. Jamneala, M. F. Crommie, and N. S. Wingreen, Phys. Rev. B 64, 165412 (2001).

${ }^{41}$ The Kondo temperature is obtained by fitting the $d I / d V$ spectra with the Fano model $\frac{d I}{d V} \propto \frac{\left(q+\varepsilon^{\prime}\right)^{2}}{1+\varepsilon^{\prime 2}}$ and $\varepsilon^{\prime}=\frac{(\varepsilon-\alpha)}{k T_{K}}$. Hereby, $\alpha$ is the energy position of the Kondo resonance and $q$ an asymmetry parameter describing the coupling to the tip wave function.

${ }^{42}$ M. Yulikov, M. Sterrer, M. Heyde, H.-P. Rust, T. Risse, H.-J. Freund, G. Pacchioni, and A. Scagnelli, Phys. Rev. Lett. 96, 146804 (2006)

${ }^{43}$ M. Sterrer, T. Risse, M. Yulikov, H.-J. Freund, J. Carrasco, F. Illas, C. Di Valentin, L. Giordano, and G. Pacchioni, Angew. Chem. 118, 2695 (2006).

${ }^{44}$ A. S. Worz, U. Heiz, F. Cinquini, and G. Pacchioni, J. Phys. Chem. B 109, 18418 (2005).

${ }^{45} \mathrm{~N}$. Quaas, M. Wenderoth, A. Weismann, R. G. Ulbrich, and K. Schönhammer, Phys. Rev. B 69, 201103(R) (2004).

${ }^{46}$ P. Kurz, G. Bihlmayer, K. Hirai, and S. Blügel, Phys. Rev. Lett. 86, 1106 (2001)

${ }^{47}$ K. von Bergmann, S. Heinze, M. Bode, E. Y. Vedmedenko, G. Bihlmayer, S. Blügel, and R. Wiesendanger, Phys. Rev. Lett. 96 167203 (2006).

${ }^{48}$ P. Ferriani, K. von Bergmann, E. Y. Vedmedenko, S. Heinze, M. Bode, M. Heide, G. Bihlmayer, S. Blügel, and R. Wiesendanger, Phys. Rev. Lett. 101, 027201 (2008). 